\title{
Investigating Cultural Macroevolution through Biological Phylogenetic Analyses of Chinese Dai People Costumes Pattern
}

\author{
Yunfeng Zhang \\ School of Life Science, Yunnan Normal University, Kunming650500, Yunnan, PRC \\ Yang Zhang \\ School of Tourism and Geographic Science, Yunnan Normal University, Kunming650500, Yunnan, PRC \\ Mingzhu Yang \\ Institute of Folk art, Yunnan art University, Kunming650500, Yunnan, PRC
}

\begin{abstract}
What are the driving forces for cultural macroevolution has engaged anthropologists for more than a century. Recently, the debate on the evolution of culture has focused on two processes in particular, phylogenesis and ethnogenesis, and has been suggested that the latter has probably always been more significant than the former. Cladistic methods of phylogenetic reconstruction was used to assess a data set consist of 119 costume motifs derived from costumes pattern and decorative characters from some groups of Dai people and its accompanying group of $\mathrm{Yi}$ and Hani people in Yunnan, China. The results indicate that phylogenesis was the dominant process in the culture evolution of costumes pattern and decorative characteristics of Dai people, accounting for about $60 \%$ of the resemblances among the Dai people costumes pattern. These results do not support the proposition that ethnogenesis has always been a more significant process in cultural evolution than phylogenesis.
\end{abstract}

KEYWORD: Dai people; phylogenesis; ethnogenesis; cladistic methods

\section{INTRODUCTION}

What are the driving forces for cultural evolution has engaged anthropologists for more than a century. Cultural macroevolution refers to the historical processes that explain cultural similarities and differences between human populations arising from the descent with modification (Mulder \& Towner 2006). At the microevolutionary scale, modern theories of cultural evolution recognize that cultural traditions and innovations are socially transmitted person-to-person between and within generations by vertical or oblique and by horizontal transmission routes, respectively (Cavalli-Sforza \& Feldman 1981),with the learners applying generalized rules in choosing when to engage in independent trial-anderror learning and selecting whose example to copy when this is the preferred strategy(Boyd \& Richerson 2010). Preservation of a historical signal within the cultural traditions carried by populations depends on the traits being consistently selected and replicated, often with some degree of modification, ensuring the culture survive from one generation to the next.

Mesoudi et al. (2006), who propose a multidisciplinary framework for the Darwinian analysis of cultural dynamics, draw an explicit parallel between evolutionary archaeology, cultural anthropology and comparative anthropology. In recent years, as has the corollary issue of linking patterns in the ethnographic and archaeological records with genetic and linguistic data, the evolution of culture is analogous to biological evolution has been considered in linguistic and material evolution (Tehrani \& Collard 2002; Ammerman \& Cavalli-Sforza 1984; Bateman et al 1990; Boyd \& Richerson 1985; Boyd et al.1997). Evolutionary approaches to cultural diversity or culture change are increasingly influential, and many scientists believe that a grand synthesis is now in sight (Mesoudi et al. 2006). A new scientific discipline, culturomics, which was introduced as the application of high-throughput data collection and analysis to the study of human culture has been formed (Gao et al.2012). And many technique, such as cladistic method, neighbor graphs analysis, and random fractal analysis were used to study culture.

In traditional cultural evolution context, the major focus in culture is the relative importance of two processes which Moore (1994) termed phylogenesis and ethnogenesis. In the former, cultural evolution is a result of the progressive subdivision of cultural assemblages that takes place as populations split and gives rise to new ones. In ethnogenesis, in contrast, cultural evolution occurs through the borrowing and blending of ideas and practices, and the trade and exchange of object, among contemporaneous populations. Cultural phylogenesis is expected to 
produce a strong association between cultural patterns and genetic and linguistic data. Ethnogenesis, on the other hand, is predicted to yield a close relationship between cultural patterns and the frequency and intensity of contact among populations, the usual proxy of which is geographic proximity (Terrell, 2001; Tehrani \& Collard 2002).

Now, it has been asserted that ethnogenesis has been the major cultural macroevolutionary process in the ethnohistorical period and is likely to have always been more significant than phylogenesis in cultural macroevolution (Moore 1994; Terrell 2001). These scientist believe that it is unrealistic to think that the history is patterned like the nodes and branches of a comparative, phylogenetic, or cladistic tree and argue that the biological, linguistic, and cultural evolution of human being is best characterized by a constant flow of people, and hence their genes, language, and culture, across the fuzzy boundaries of tribes and nations, spreading within a region(Terrell 2001). However, this assertion is open to a question as the debate on the relative importance of phylogenesis or ethnogenesis, and there is a pressing need for further empirical assessments of the issue. The present proposal describes a case study in which biological phylogenetic methods were applied to cultural data with a view to assert the relative importance of phylogenesis and ethnogenesis in cultural macroevolution of Dai people's costumes pattern.

\section{CASE STUDY: DAI PEOPLE GROUP CUSTOME}

The case study dealt with the macroevolution of culture among different group of Dai people in Yunnan, China, with their accompanying ethnic group Yi and Hani people as control. Dai people are one of the 56 ethnic groups officially recognized by the People's Republic of China. Dai people is one of several ethnic groups accompanying with other ethnic group (e.g. Yi, Hani, Buyi, et al) living in southern Yunnan, China, but by extension can apply to groups in Laos, Vietnam, Thailand, and Burma when Dai is used to mean specifically Tai Lue, Chinese Shan, Tai Dam, Tai Khao or even Tai in general. Originally, the Tai or Dai, lived closely together in modern Yunnan until political chaos and wars in the north at the end of the Tang and Song Dynasty and various nomadic peoples prompted some to move further south into modern Laos then Thailand (Zhu 1992).

Although they are officially recognized as a single people by the Chinese state, Dai people form several distinct cultural and linguistic groups. In linguistic context, there are four main languages, Dai Lü (Sibsongbanna Dai), Dai Nüa (Daihong Dai), Tày Pong, and Tai Dam in written languages, belong to difference language branch in Tai-Kadai language family(Sagart 2004).Beyond that, many other groups, especially who live at central part of Yunnan, have no written language. In religion context, Dai people divided into two groups, one has their traditional religion as well as Theravadin Buddhism, and the other one only has their traditional religion, namely adhere to the animism ( $\mathrm{Qu}$ 2012). Both groups maintain similar customs and festivals to the other Tai-speaking peoples with little difference. In demography context, there are two group of Dai people, one's population is large and dwell together in the same region, such as Xishuangbanna Dai autonomous Prefecture (shorted as Banna in the following) and Dehong Dai and Jingpo autonomous Prefecture (shorted as Dehong in the following) along Nujiang River and Lancangjiang River (Mekong river); another population is small, live scattered at many sites alone Yuanjiang River (called Honghe after enter Vietnam). Some group of later population who live along AiLaoShan mountain is called as Huayao Dai, named for having multicoloured waistband in its costumes of women. Although, there is debate on this appellation, the named Huayao Dai is very popular presently (Qu 2012). Many anthropologists argue that Huayao Dai is an appellation named by other people, scientific appellation should be the name of themselves (Li 2009). Huayao Dai, especially who live at Xingping and Yuanjiang in Yunan, has been regarded as royal lineage descendant of ancient Dian Kingdom, a state existed from early Warring States to Eastern Han Dynasty (500BC-100AD) in China history, because of many archaeological artifacts such as bronze wares and bronze drums which were characteristics of ancient Dian Kingdom were discovered in the sites near their living place (Suen, 2006). Huayao Dai are considered to be left in the Yuanjiang river valley during the migration of Dai people in history after invaded from other races. Huayao Dai divide into many group according to their self name, such as Daisa, Daika, Daiya, Daide,et al, nomenclature following name of their living place, legend story or career. Those groups has no write language, and have their own culture characteristic compare to Dai Lü and Dai Nüa, and is regarded as keeping the important districts native culture element of Dai culture. They are no influence Theravadin Buddhism culture on the people of those groups, and are considered to preserve completely the primitive culture characteristic of ancient Dai ancestor, such as worship, witchcraft, dye teeth, tattoos. And their costumes pattern are considered to reflect the most distinctive of Dai native cultural characteristics, in spite of the traditional attires are only worn during certain festivals, ceremonies or religious occasions nowadays. 
The costumes pattern varied in different groups of Dai, Yi, and Hani people. The poor preservation of old sample and absence in write recording over time, means that very little is known about its origins and development. In fact, the whole history of ancient Dian kingdom and ancient Dai people have been lost in written-record after Han Dynasty (206BC-220A D).The costume pattern evolution of Dai people is a classic example of the Problem of Missing Links. It is likely that costumes were developed by their ancestor, which may be found evidence in archaeological material excavated in Yuanjiang River basin (Suen 2006). Each group of Dai people has its own distinctive costume pattern, although many parts and decorate are shared by different groups. It has often been claimed that these similarities can be traced back to common ancestral group (e.g.Dai ancestor of ancient Dian kingdom, Suen 2006). However, this hypothesis needs to be tested against the alternative possibility that contact and exchange among groups might have led to borrowing and blending among their costume traditions.

Dai people costumes patterns are an especially interesting context for investigating the phylogenesis and ethnogenesis problem because of the ways in which craft knowledge is transmitted among Dai people woman, such as mother to daughters or other older women to younger one. From 2012, we visit more than 10 times to various Dai people group inhabited sites in Yunnan and interviewed over 20 women about how they learned to tailor. Figure 1 is The Geographic distribution of some groups of Dai people and its accompanying ethnic group $\mathrm{Yi}$ and Hani at XingPing and Yuanjiang, where we investigated the costumes. The interviews revealed important differences in the ways that techniques and designs are transmitted. During their apprenticeship, young tailor also build up a repertoire of designs by collaborating with and imitating their mothers or other older women. However, whereas adult tailors rarely acquire new techniques once they begin to work independently, they frequently copy designs from their peers. We can therefore hypothesize that while the transmission of tailoring techniques follow similar pathways to the transmission of genes (i.e. they are transmitted vertically between generations), the transmission of designs is likely to be much more complicated (since they can be transmitted vertically between generations and horizontally within generations).

To test this hypothesis, we used a phylogenetic method known as cladistic analysisn, a method has been used widely in linguistic data and material culture (Matthews et al., 2011; Whiten et al., 2011; Tehrani et al.2010; Greenhill et al.2010; Steele et al., 2010; Shennan 2009; Temkin\&Eldredge 2007; Collard et al.2006; Tehrani\& Collard 2002;O’Brien et al.,2001; Gray \&Jordan2000;). The cladistic analysis focuses on variation in the constituent parts, or characters of a group of taxa. In these studies the equivalent of a biological species or taxon is the culture or language under consideration. In biological species, characters may comprise DNA sequences or morphological traits. In languages, characters are usually based on lists of words, such as core vocabulary items. In the case of material culture, characters consist of stylistic and/or technological elements of assemblages, such as aspects of arrowhead design (O'Brien \& Lyman 2003), forms of musical instruments (Temkin \& Eldredge 2007), motif of textile (Tehrani\& Collard 2002) or, in our case, pattern variations in costumes constitute and its ornaments, which we called as costumes motif. Figure 1 is some costume samples and head ornaments comparison in different groups in Xinping.

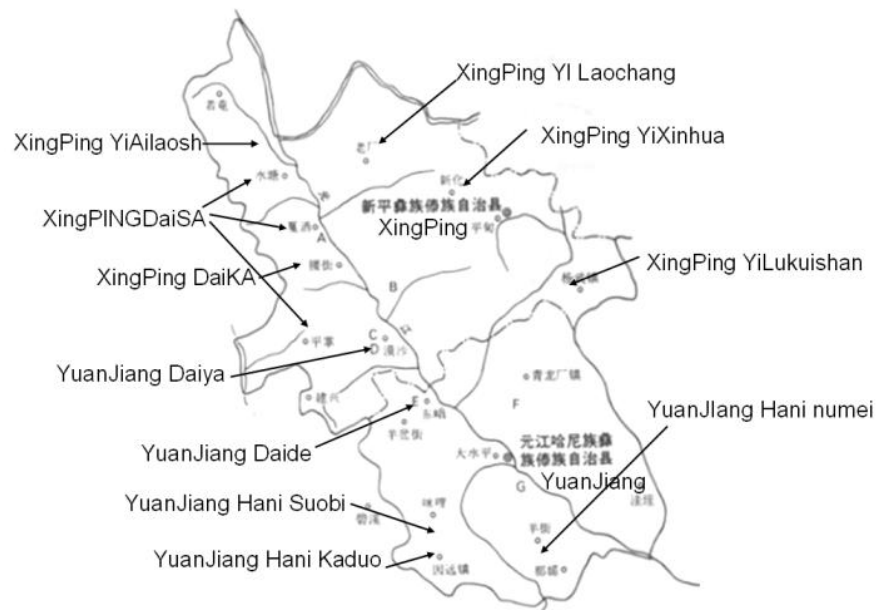

Figure 1 The Geographic distribution of Dai people and its accompanying ethnic group $\mathrm{Yi}$ and Hani at Xingping and Yuanjiang (after Zhen, 2001)

The case study employed 119 characters (costumes motif) in 37 ethnic groups, among them, there are 15 Dai groups, 8 Yi groups and 4 Hani groups, respectively. These selected Yi and Hani groups are living in same region with Dai people in XingPing and Yuanjiang. The costumes motif include upper garment pattern, lower garment pattern, collars pattern, sleeves pattern, buttons pattern, headwear pattern, and their ornament and concerning material. For example, for upper garment motif, all of the information concerning the upper garment is considered, such as, the short or long for garment length according whether they arrive navel (short upper garment which only cover chest of body, is a characteristics for some Dai people groups), the short or long for sleeves, having collar or not, right laple or left laple, having garment front or not, et al. Figure 2 is some costume samples and head ornaments comparison in various groups. All costumes motifs were then defined in such a way that they could be scored as either present or absent. 
Once the data were coded, we construct a matrix in which the taxa were listed in the row headings and the motif listed in the column headings. The analyses were carried out by MEGA4.0 (Tamura et al., 2007) with the coded $G$ or $C$ for the motif present or absent (because the MEGA software can only distinguish the symbol of DNA or abbreviation of protein) and calculate concerning parameters with same software.

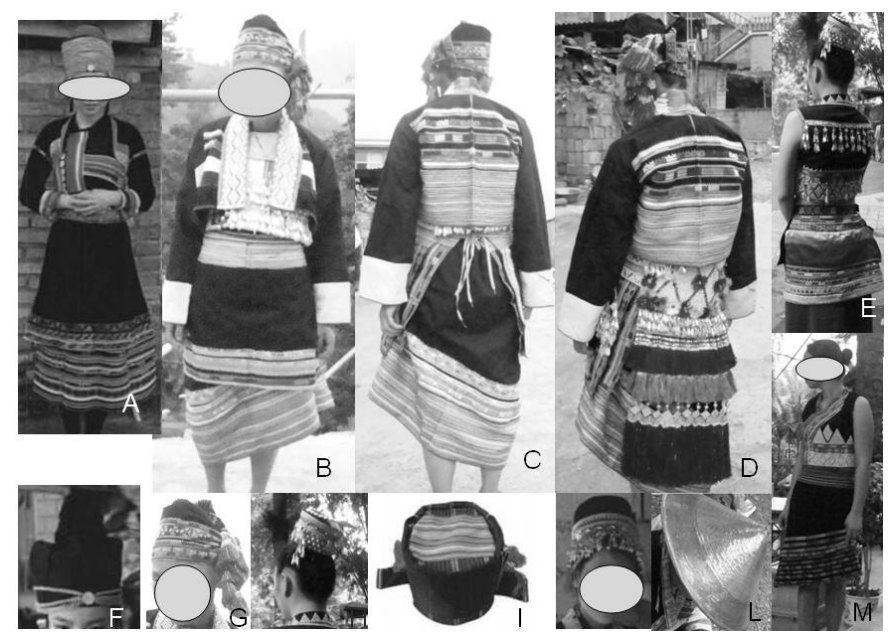

Figure2 Some costumes samples and head ornaments comparison in various groups. The groups name is annotated toponymy+ethnic group name. A. Banna Daiya (young woman); B. Xingping Daika(old woman at front); C. Xingping Daika(old oman at back); D. Xingp Daika (old woman at back with tail decorations; E. Xingping Daisa(young woman at back); F. Banna Daiya(Adult women); G. Yuanjiang Daika(old woman); H. Xingping Daiya(young woman); I. BannaDaiya (old woman); J. Yuanjiang Daisa(young woman); L. Xingping Daiya (old woman with bamboo hat); M. Xingping Daisa (young woman). All of the faces of models were cover by a roundel.

\section{RESULTS}

The costumes matrix which had coded was used to analyze via MEGA4.0 software, the yielded cladistic tree was shown in Fig 3. The tree was inferred using the Maximum Parsimony method (Eck \& Dayhoff 1966), and was selected out from 11 most parsimonious trees (length $=301$ ). The consistency index, retention index, and composite index is 0.348837 (0.312281), 0.485564 (0.485564), and 0.169383 (0.151632) for all sites and parsimonyinformative sites (in parentheses). The MP tree was obtained using the Close-Neighbor-Interchange algorithm (Nei\& Kumar 2000) with search level 2 in which the initial trees were obtained with the random addition of sequences (1000 replicates). There were a total of 112 positions in the final dataset with 89 were parsimony informative.

From the Figure 3 we can found that the costumes culture of the Dai,Yi,and Hani people is clustered into one group, respectively,in spite of with exception in Yuanjiang Daile and Yuanjiang YiLamei, and Yuanjiang HaniKadou.That suggested that ethnic costumes of Dai, Yi and Hani people are descended from their own common ancestor in general, respectively. And Dai people divided further into two branchs, the first branch include Dai people group in Xingping, Yuanjiang, Dayao and Banna, the second branch include Dai people group in Jingping, Dehong. It is very interesting that Menlian Dai is out from the two branchs. More detail in the cladogram, we could found that Banna Mengyang Daiya and Xingping Daisa, Banna Dailue and Dayao Dai, Jingping Dailuo and Jingping Daiuudainan, are clustered into a sub-branch, respectively. In geography, the distance between Banna Mengyang and Xingping or Banna and Dayao are longer than that of Xingping and yuanjiang, also suggest that phylogenesis has been a more significant process than ethnogenesis in Dai costumes pattern evolution.

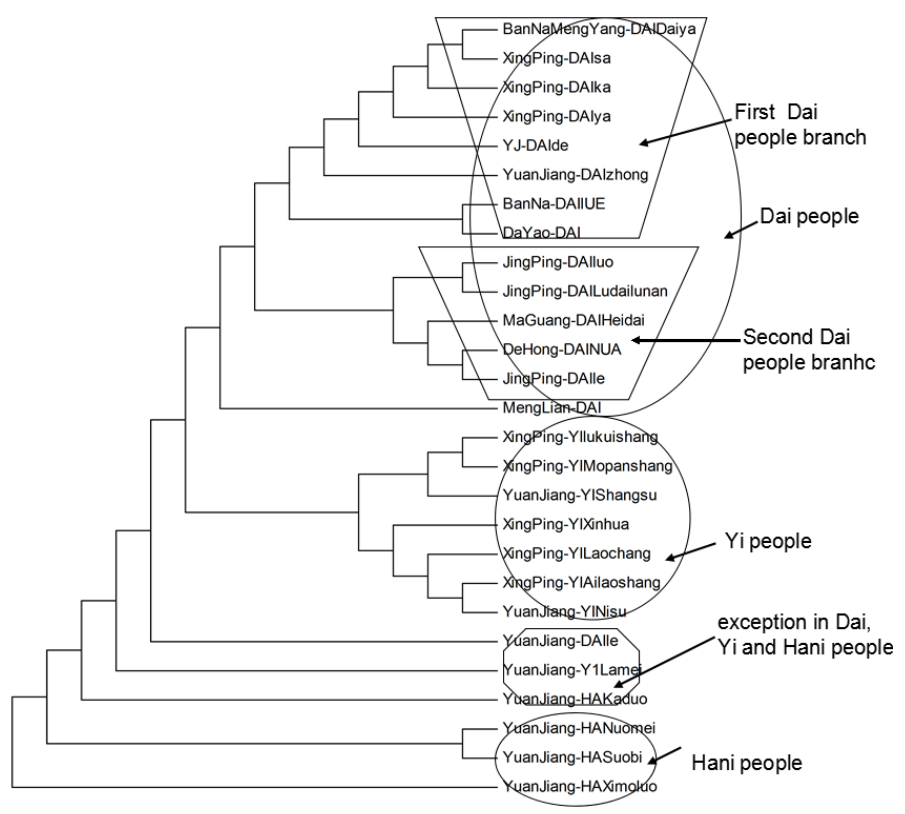

Figure 3 Most parsimonious cladogram obtained in the analysis designed to determine the relative contributions of phylogenesis and ethnogenesis to the costumes evolution of Dai people and its accompanying ethnic groups in Yunnan. The name of groups is annotated toponymy+ethnic group name.

\section{DISCUSSION}

The results of these analyses suggest that the majority of similarities among the costumes pattern assemblages of Dai people and its accompanying Yi and Hani people can be explained by a tree-like model of descent with modification with their own ancestor. The fit between the data and the cladogram was assessed with the Consistency Index (CI) and retention index (RI) with bootstrapping.

The CI and RI is a measure of how parsimonious evolution has been for a given combination of cladogram and data set, i.e., it is a measure of the number of homoplasies in a data set (Kitching et al., 1998). A CI or RI of 1 indicates that the data are perfectly congruent with the cladogram. When 
compare CI or RI with other different studies in the cladistic analysis, we find the CI and RI in our case study is too low, in other case the CI values almost above 5.0(Tehrani \& Collard 2002; Chamber-lain \& Wood, 1987; Lieber-man et al. 1996; Strait et al. 1997). The reason for the contradiction, we think it is maby for data sourcing we selection. Yi and Hani people belong to ancient Qiang people, another big ethnic group. In tradition, their costumes have own characteristic, and were different from that of Dai people greatly (belong to another big ethnic group, Baiyue). Therefore, we remove the data from costumes motif matrix, calculate again the $\mathrm{CI}$ and RI parameters using MEGA4.0. By this time, the CI and RI is 0.582558 and 0.570238 , respectively. And the parsimonious tree was shown in Figure 4.The result is very similar to that of Turkmen textile design (0.68) (Tehrani \& Collard 2002). The CI and RI of the bootstrap analysis suggest that ethnogenesis was considerably less important than cultural phylogenesis. In biology, the value of CI and RI is about 0.5 means the character is neutral during evolution.In fact, when ethnic group select their costumes pattern, the costumes pattern will not have great influence to their surviving, and variation are also exist intra-group and difference stage of her life.

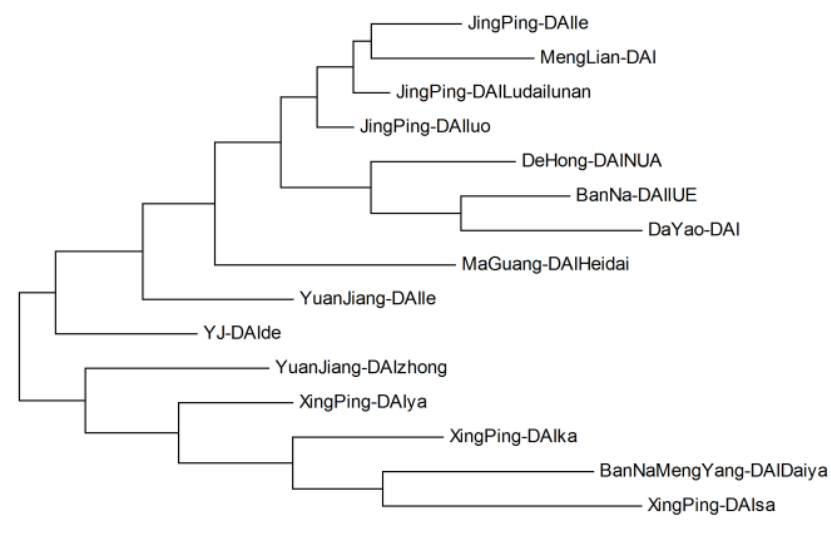

Figure 4 Most parsimonious cladogram obtained in the analysis designed to determine the relative contributions of phylogenesis and ethnogenesis to the costumes evolution of Dai people. Annotation is same as in Figure 3.

According to the $\mathrm{CI}$ or RI for the cladogram (0.58, 0.57, respectively), about $60 \%$ of the similarities among the assemblages are homologous and approximately $40 \%$ are homoplastic. This is compatible with the borrowing of costumes motifs being responsible for a third of interassemblage resemblances, although the possibility of independent invention as a source of homoplastic similarities cannot be completely discounted. Regardless of the source of the homoplasies, it is clear that phylogenesis was the dominant cultural evolutionary process among the Dai people costumes. And the results are also same for $\mathrm{Yi}$ and Hani people (data not shown).

Cladistic analyses were also carried out to ascertain whether the relative contributions of phylogenesis and ethnogenesis to the evolution of Dai people costumes altered following living with other people. The CI and RI decrease $33 \%$ and $16 \%$, respectively. The results indicated that the social changes experienced by cohabitation led to a greater role for ethnogenesis in Dai people costumes cultural evolution. Phylogenesis remained the dominant cultural evolutionary process, but the importance of ethnogenesis increased after fusion with other people. Thus, there is about 33\% increase in the number of homoplastic resemblances among the costumes assemblages after cohabitation. This is consistent with more intertribal borrowing of designs and motifs, but again independent invention cannot be entirely discounted as a source of homoplasies. If the homoplasies are assumed to result mainly from diffusion, then the analyses indicate that $\mathrm{Yi}$ people costumes were a particularly important source of designs and motifs for Dai people than that of Hani people. This deduction is corresponding to fact in history. After Yuan Dynasty, time when the Dai people costumes had change greatly, the Yi people gradually became dominate ethnic group at many place in Yunnan, such as Xingping, Yuanjiang and Jingping et al, with the policy of bureaucratization of native officers offered by central government. Which was called as conformism, a mechanisms affect culture macroevolution (Boyd \& Richerson 2010). These phenomena can be found from the cladogram in Figure 3 and Figure4. The Jingping Dailuo, MaguanHeiDai, Yuanjiang Daode et al, their costume pattern is not cluster into other Dai people branch. In investigation of costumes, their costumes had changed more or less like their accompanying people. Jingping Daoluo have flower embroider at front of garment, and garment became losing like Yi and Hani people, Maguan Hei Dai had more headwear like Zhuan people; Yuanjiang Daide abandon Baiyue's symbol-tube skirt, wear trousers like Yi and Hani people. Of course, this is first time to investigate the cultural macroevolution through biological phylogenetic analyses in Chinese ethnic costumes pattern, the chosen motif may be not express full information of an ethnic costumes, more detail motif and other information are needed for further study.

\section{ACKNOWLEDGEMENTS}

We are grateful to Prof. Dr. Li Yong Xiang of Yunnan academy of social sciences for helpful discussions about ethnic group and ethnic costumes. We also thank "Philosophy and social sciences 
planning project of Yunnan Province" (YB2012037) for financial support to this project.

\section{REFERENCES}

[1] Ammerman A.J.\&Cavalli-Sforza L.L.1984. The Neolithic Transition and the Genetics of Populations in Europe. Princeton University Press, Princeton.

[2] Bateman, R., Goddard, I., Grady, R., Funk, V.A., Mooi, R., Kress, W.J., Cannell, P., 1990. Speaking with forked tongues: the feasibility of reconciling human phylogeny and the history of language. Current Anthropology 31, 124.

[3] Boyd, R. \& Richerson, P., 1985. Culture and the Evolutionary Process. University of Chicago Press, Chicago.

[4] Boyd R.\&Richerson P. J. 2010 Transmission coupling mechanisms: cultural group selection. Phil. Trans. R. Soc. B 365, 3787-3795.

[5] Boyd, R., Borgerhoff Mulder, M., Durham, W.H., Richerson, P.J., 1997. Are cultural phylogenies possible? In: Weingart, P., Mitchell, S.D., Richerson, P.J., Maasen, S. (Eds.), Human by Nature. Lawrence Erlbaum, Mahwah, pp. 355-386.

[6] Cavalli-Sforza L. L.\&Feldman, M. W. 1981 Cultural transmission and evolution. Princeton, NJ: Princeton University Press.

[7] Chamberlain, A.T.\&Wood, B.A., 1987. Early hominid phylogeny. Journal of Human Evolution 16, 119-133.

[8] Collard M, Shennan SJ, Tehrani J.J. 2006. Branching, blending and the evolution of cultural similarities and differences among human populations. Evol Hum Behav 27: $169-184$

[9] Eck RV \& Dayhoff MO.1966.Atlas of Protein Sequence and Structure. National Biomedical Research Foundation, Silver Springs, Maryland.

[10] Farris J.S.1989. The retention index and homoplasy excess. Syst Zool. 38:406-7.

[11] Gray R.D. \& Jordan.FM.2000. Language trees support the express-train sequence of Austronesian expansion. Nature 405 1052-1055.

[12] Greenhill SJ, Drummond AJ, Gray RD. 2010. How accurate and robust are the phylogenetic estimates of Austronesian language relationships? PloS ONE 5(3): e9573.

[13] Gao J.B., Hu J., Mao X., and Matjaz Perc.2012. Culturomics meets random fractal theory: insights into long-range correlations of social and natural phenomena over the past two centuries. J. R. Soc. Interface 9, 19561964.

[14] Kluge, A.Wolf, 1993. Cladistics: what $\mathrm{s}$ in a word? Cladistics 9, 183-199.

[15] Li yong-Xiang.2009.Ethnic symbolism and ethnic relation of the Daiya costume. Journal of Bijie University, 27(9):92-97.

[16] Lieberman, D.E., Wood, B.A., Pilbeam, D.R., 1996.Homoplasy and early Homo: an analysis of theevolutionary relationships of $H$. habilis sensustric to and $H$. rudolfensis. Journal of Human Evolution 30, $97-$ 120.

[17] Matthews LJ, Tehrani JJ, Jordan FM, Collard M, Nunn CL. 2011.Testing for divergent transmission histories among cultural characters: a study using Bayesian phylogenetic methods and Iranian tribal textile data. PLoS ONE 6(4):e14810.

[18] Mesoudi A., Whiten, A. \& Laland, K. N. 2006. Towards a unified science of cultural evolution. Behav. Brain Sci.29, 329-383.

[19] Moore, J.H.1994. Ethnogenetic theory. National Geographic Research and Exploration 10, 10-23.

[20] Mulder, M. B., Nunn, C. L. \& Towner, M. C. 2006 Cultural macroevolution and the transmission of traits. Evol. Anthropol. 15, 52-64

[21] Nei M \& Kumar S. 2000. Molecular Evolution and Phylogenetics. Oxford University Press, New York.

[22] Nunn CL, Arnold C, Matthews L, Borgerhoff Mulder M. Simulating trait evolution for cross-cultural comparison. Philos Trans R Soc B. 2010; 365:3807-21.

[23] O’Brien MJ, Darwent J, Lyman RL. 2001. Cladistics is useful for reconstructing archaeological phylogenies: Paleoindian points from the southeastern United States. J Archaeol Sci. 28:1115-36.

[24] Qu Yong-xian.2012.South-east Tai-Thai Ethnic Culture and its Naming Evolution Journal of Guanxi Normal University for nationalities, 29(6):39-43.

[25] Sagart, L. 2004. The higher phylogeny of Austronesian and the position of Tai-Kadai. Oceanic Linguistics 43.411-440.

[26] Sanderson, M.J.1995. Objections to bootstrapping phylogenies: a critique. Systematic Biology 44, 299-320.

[27] Shennan S. 2009. Pattern and process in cultural evolution. London, UK:University of California Press.

[28] Steele J, Jordan P, Cochrane E. 2010. Evolutionary approaches to cultural and linguistic diversity. Phil. Trans. R. Soc. B 2010 365, 3781-3785.

[29] Strait, D.S., Grine, F.E., Moniz, M.A., 1997. A reappraisalof early hominid phylogeny. Journal of Human Evolution 32, 17-82.

[30] Suen Jun.2006.HuaYao Dai relationship with the origin and development of ancient dian kingdom. In:The collection of international academic seminar on the culture of Hua yao Dai, Xingping, Yunan, China. Bejing, Ethnic Press.

[31] Tamura K, Dudley J, Nei M \& Kumar S.2007.MEGA4: Molecular Evolutionary Genetics Analysis (MEGA) software version 4.0. Molecular Biology and Evolution 24:1596-1599.

[32] Tehrani,J. \& Collard.M. 2002. Investigating cultural evolution through biological phylogenetic analyses of Turkmen textiles. Journal of Anthropological Archaeology, 21:443-463.

[33] Tehrani JJ, Collard M, Shennan SJ.2010.The cophylogeny of populations and cultures: reconstructing the evolution of Iranian tribal craft traditions using trees and jungles. Philosophical Transactions of the Royal Society B: Biological Sciences 365: 3865.

[34] Temkin I \& Eldredge N.2007. Phylogenetics and material culture evolution. Curr Anthropol. 48:146-53.

[35] Terrell, J.E. 2001. Introduction. In: Terrell, J.E. (Ed.), Archaeology, Language, and History: Essays on Culture and Ethnicity. Bergin and Garvey, Wesport,pp. 1-10

[36] Whiten A, Hinde RA, Laland KN, Stringer CB.2011.Culture evolves. Phil.Trans. R. Soc. B 2011 366, 938-948.

[37] Zheng Xiao yuan. 2001. The culture of Huayao Dai and its evolution. Journal of Yunnan social sciences.2:59-64.

[38] Zhu Liangwen.1992. The Dai: Or the Tai and Their Architecture \& Customs in South China. Bangkok, Thailand, and Kunming, Yunnan, China: D Books and The Science and Technology Press of Yunnan 